\title{
Multi-Packet Reception Method for Neighbor Discovery in Wireless Network
}

\author{
Chaya P \\ Assistant Professor, Department of Information Science and Engineering, GSSS Institute of Engineering and
}

Technology for Women, Mysore, India

\begin{abstract}
Neighbor discovery is one of the first steps in configuring and managing a wireless network. Most existing studies on neighbor discovery assume a single-packet reception model where only a single packet can be received successfully at a receiver. Neighbor discovery in MPR networks is studied that allow packets from multiple simultaneous transmitters to be received successfully at a receiver. Starting with a clique of $n$ nodes, a simple Alohalike algorithm is analyzed and shows that it takes time to discover all neighbors with high probability when allowing up to $\mathrm{k}$ simultaneous transmissions. Two adaptive neighbor discovery algorithms is designed that dynamically adjust the transmission probability for each node. The friend algorithms yield improvement over the Aloha-like scheme for a clique with $\mathrm{n}$ nodes and are thus order-optimal.
\end{abstract}

Keywords: Wireless Networks, Multi packet Reception, Network Management, Neighbor Discovery, Friend Algorithm.

\section{INTRODUCTION}

A wireless network is any type of computer network that uses wireless data connections for connecting network nodes. Wireless networking[1] is a method by which homes, telecommunications networks and enterprise (business)installations avoid the costly process of introducing cables into a building, or as a connect ion between various equipment locations. Wireless telecommunications networks are generally implemented and administered using radio communication. The Neighbor discovery is one of the first steps in configuring and managing a wireless network. The information obtained from neighbor discovery, viz. the set of nodes that a wireless node can directly communicate with, is needed to support basic functionalities such as medium access and routing.Furthermore, this information is needed by topologycontrol and clustering algorithms to improve network Performance. Due to its critical importance, neighbor discovery has received significant attention, and a number of studies have been devoted to this topic Most studies, however, assume a single packet reception (SPR) model, This is motivated by the increasing prevalence of MPR technologies in wireless networks. First we analysis the Aloha-like neighbor discovery algorithm, and show that the neighbor discovery time is $\mathrm{T}(\ln \mathrm{n})$ in an idealized MPR network that allows an arbitrary number of nodes to transmit simultaneously, and the neighbor discovery time is $\mathrm{T}(\mathrm{n} \ln \mathrm{n} \mathrm{k})$ when allowing up to $\mathrm{k}$ nodes to transmit simultaneously. In this paper to propose two adaptive neighbor discovery algorithms, one being collisiondetection[9] based, and the other being ID based. In both algorithms, a node becomes inactive once it is discovered by its neighbors, allowing there active nodes to increase their transmission probability.

\section{PROBLEM STATEMENT}
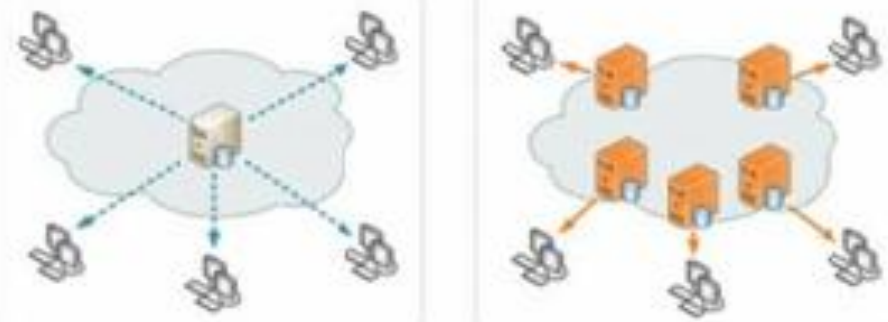

Fig1: Multipacket reception

We design two adaptive neighbor discovery schemes that improve upon the Aloha-like scheme. Both schemes utilize feedback information from nodes to achieve faster discovery. One of the schemes requires collision detection[9] at nodes, i.e., the ability to distinguish between a collision and an idle slot, while the other scheme only requires each 


\section{A. Adaptive Neighbor Discovery}

The motivation for an adaptive neighbor discovery algorithm is to get maximal service availability with minimum bandwidth consumption for a given error rate in different wireless network environments[1]. Nodes may move with totally random speed and movement patterns.

\section{B. Collision Detection-Based Algorithm.}

Collision Detection based Neighbor Discovery [9], a node uses the collision based neighbor discovery algorithm to know whether its transmission is successful or not. Now we assume the node can distinguish between a collision and an idle slot. We divide a slot into two sub slots. In first slot the odes either transmit or listen the packets. If a node listens in the first sub-slot and can decode the received packets successfully, then it deterministically sends a signal in the second sub-slot; otherwise, it remains silent. A node that transmits in the first sub-slot knows its transmission is successful if and only if it hears a signal (or senses energy) in the second sub-slot.

\section{Id Based Neighbor Discovery}

ID-based scheme, we require each node to record the IDs of the nodes that it hears in each slot. When a node transmits, it transmits its ID as well as the IDs of every node from which it successfully received a message in any of the past slots. The key challenge in the ID-based feedback scheme is in devising an efficient scheme to encode node IDs in the messages transmitted by nodes to ensure that the message lengths remain bounded. A naive implementation of the IDbased feedback scheme in which each node uses the binary representation of the IDs, can lead to very long message lengths.

\section{D.FRIEND PROTOCOL}

\section{HD-Friend: Friend for Half-Duplex:}

With HD-FRIEND[3] half-duplex networks the time required for neighbour discovery process also get decreased.There should be one more subslot that is used for transmitting feedback signals, because radios are half duplex and cannot notify collisions during reception. As a result, there are three subslotsin one iteration. The first subslot is used to conduct the greeting process (GR subslot), and the second subslot is used for transmission of the discovery message (TR subslot). Feedback signals are transmitted in the third subslot (FB subslot).

HD-FRIEND-GR[3] (Half Duplex)

If a node intends to transmit in TR, it will send Ms in GR to notify other nodes and send Md in TR, regardless of other nodes actions. Receiving nodes behave the same way as they are in FRIEND Algorithm.

HD-FRIEND-TR[4] (Half Duplex)

For a transmitting node, it will send its discovery message during TR and keep listening in FB to get feedback. While for a receiving node, it will keep listening in TR to determine whether to send a feedback signal to notify the transmitting nodes of the failure in transmission. After TR, nodes enter the FB subslot, and the transmitting node will be aware of whether its transmission is successful.

HD-FRIEND-FB[4] (Half Duplex)

In the FB subslot, if a receiving node detects collision, it will broadcast a feedback signal. As a result, transmitting nodes know that their transmissions failed. On the other hand, if the transmitting node does not receive the feedback signal, it knows that the transmission is successful, and it is time to keep silent during the remaining process of ND.

FRIEND protocol[5] does its neighbour discovery by adding pre hand shaking sub slots before the traditional slots; also it applies full-duplex technology and used it to conduct prehandshaking with new feedback mechanisms.

\section{RELATED WORK}

A multiuser-detection based approach is used for neighbor discovery. They require each node to possess a signature and Further, nodes are assumed to operate in a synchronous manner[1]. Three power-saving protocols to schedule asynchronous node wake-up times in multi-hop networks, and describe deterministic neighbor discovery schemes in each of the three protocols[2]. Birthday-like randomized neighbor discovery algorithms that require synchronization among nodes[3].

\section{METHODS}

The algorithms proposed to use a multiuser-detection based approach for neighbor discovery. They require each node to possess a signature as well as know the signatures of all the other nodes in the network.

The main methods adopted in this project are:

1. Pre-Handshaking(FRIEND-GR)

It is the process that takes place to establish the rules of communication when communicating with a target node. After this the two nodes send several messages back and forth that enable them to agree on a communication protocol. 
2. Neighbor Discovery(FRIEND-TR)

It is a process of discovering the neighboring nodes in the path to the destination node.It also keeps track of the collision awareness of the nodes and determines the initialization and termination criteria of the nodes.

3. Multiple Pre-handshaking (FRIEND TGR)

The objective of this process is to initiate connection with the target node. The source node sends data packets over the network and the target node must have open ports that can accept and initiate connections.

4. Half Duplex

Half duplex connection is used since the data can be transmitted in both the directions but not at the same time.Here,the transmission and acknowledgement of packets cannot be done at the same time. When source node finishes transmitting packets,target node sends acknowledgement.

\section{RESULTS AND DISCUSSION}

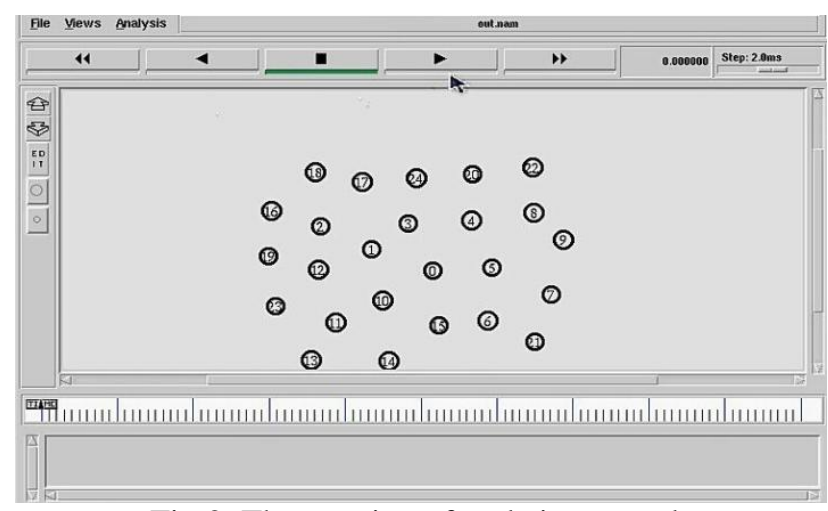

Fig 2: The creation of node in network

Establishing the connection between source and the destination node. A node can transmit simultaneously with several other neighbors, and each of these nodes may be discovered simultaneously by the receiving nodes.

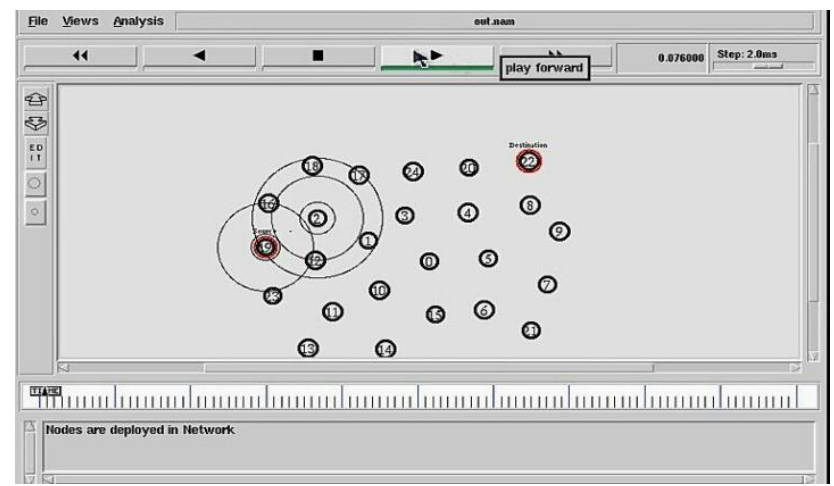

Fig 3: Finding the shortest path between node 19 to 22

Nodes are deployed which minimize energy consumption and can extend the lifetime of wireless networks

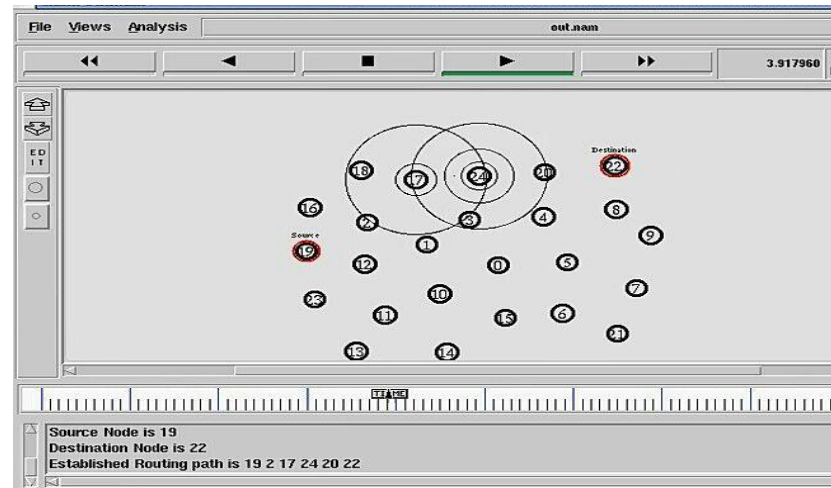

Fig 4Establishing the feasible path to node 22 
The source node 19 establishes a feasible path through the nodes $2,17,24,20,22$ to reach the destination node 22 .

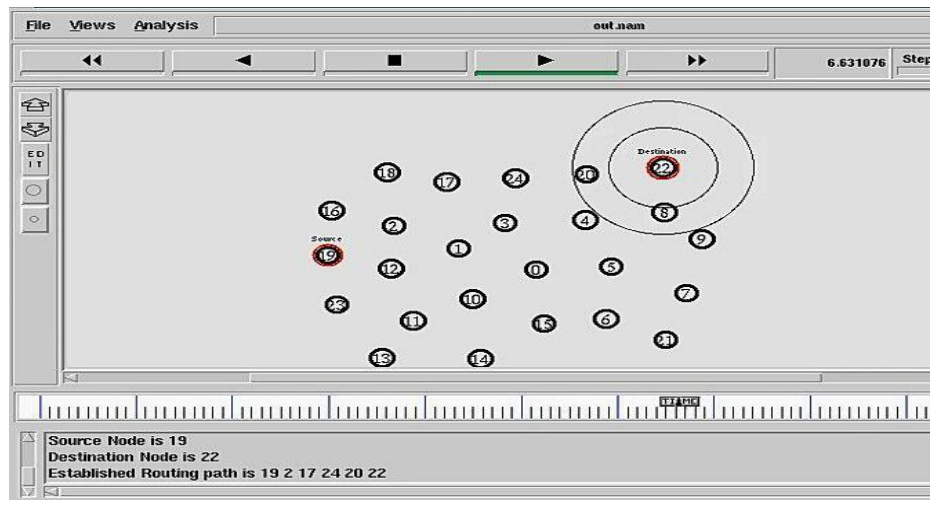

Fig5: Packet reached to the destination

Packets are at node 20 and have traversed all the other nodes from the source using the shortest path to reach the destination node. Packets are transmitted from the source node 19 to the destination node 22.

\section{CONCLUSION}

In this paper, we designed and analyzed randomized algorithms for neighbor discovery for both clique and general network topologies. The Aloha-like algorithm has scenarios such as when the number of neighbors is not known beforehand and the nodes are allowed to transmit asynchronously which is named by two adaptive algorithm. Finally We show that the FRIEND protocol and adaptive algorithms yield a $T(\ln n)$ improvement over the Aloha-like.

In most of the existing protocol have no multiple packet reception is used that is, a collision occurs when two or more nodes simultaneously transmit packets to it in a slot. So in our work we have developed the FRIEND protocol conducts the neighbor discovery process and it significantly reduces the probability of generating idle slot, collisions and enhancing network lifetime.

\section{REFERENCES}

[1] Angelosante.D, Biglieri.E, andLops.M, Neighbor discovery in wireless networks: a multiuser-detection approach. In Information Theory and Applications Workshop, pages 46-53, February 2007.

[2] Tseng et al. propose three power-saving protocols to schedule asynchronous node wake-up times in IEEE 802.11 MobiCom, August, 2000.

[3] Borbash.S.A, Ephremides.A, and McGlynn.M.J.An asynchronous neighbor discovery algorithm for wireless sensor networks. Ad Hoc Networks, 5(7):998-1016, 2007.

[4] Balakrishnan.H, Chandrakasan.A.P, Heinzelman.W.BAn application-specific protocol architecture for wireless micro sensor networks and IEEE Trans. Wireless Communications, 1(4), October 2002.

[5] Cohen.Band Kapchits.R, Continuous neighbor discovery in asynchronous sensor networks. IEEE/ACM Trans.on Networking, 19(1):69 -79, February 2011.

[6] Goeckel.D, Khalili.R ,Towsley.D, and A. Swami. Neighbor discovery with reception status feedback to transmitters. In Proc. of IEEE INFOCOM, March 2010

[7] Hannikainen.M, Kohvakka.M, Kuorilehto.M, Kaseva. and T. D. Hamalainen. Energy-efficient neighbor discovery protocol for mobile wireless sensor networks. Ad Hoc Networks, 7(24), January2009

[8] Keshavarzian.A,.V, Herrmann.F, Manjeshwar.A, and Uysal-Biyikoglu .D Energy-efficient link assessment in wireless sensor networks. In Proc. of IEEE INFOCOM, March 2004.

[9] Lin.D.D and Lim.T.J Subspace-based active user identification for a collision-free slotted ad hoc network. IEEE Transactions on Communications, 52(4):612-621, April 2004.

[10] Rus.D and Li.Q, "Sending messages to mobile users in disconnected ad-hoc wireless networks," Proc. of ACM. 